\title{
Charakterisierung eines Elektroneneinfangdetektors mit nicht-radioaktiver Elektronenquelle
}

\author{
Erik Bunert*, Ansgar T. Kirk, Maria Allers, Jens Oermann, Niklas Deutsch, Stefan Zimmermann \\ Leibniz Universität Hannover, Institut für Grundlagen der Elektrotechnik und Messtechnik, \\ Fachgebiet Sensorik und Messtechnik, Appelstr. 9A, 30167 Hannover, Deutschland \\ *E-Mail: bunert@geml.uni-hannover.de
}

\begin{abstract}
Zusammenfassung
Elektroneneinfangdetektoren (ECD) werden häufig allein oder in Kombination mit der Gaschromatographie (GC) für den schnellen Nachweis oder die Analyse von elektronenaffinen Substanzen eingesetzt. Elektroneneinfangdetektoren sind dabei die empfindlichsten Detektoren, die für solche Verbindungen erhältlich sind. Aufgrund ihres Wirkprinzips benötigen sie freie Elektronen bei Atmosphärendruck, die in der Regel durch einen $\beta$-Zerfall erzeugt werden. Die Verwendung radioaktiver Stoffe führt jedoch zu regulatorischen Beschränkungen bei Anschaffung, Betrieb und Entsorgung. Diese Arbeit zeigt grundlegende Untersuchungen zur Funktionsweise eines neuen Elektroneneinfangdetektors mit einer nicht-radioaktiven Elektronenquelle. Dieser neuartige Detektor unterliegt keinen Beschränkungen und bietet weitere Vorteile wie einstellbare Elektronendichten und energien. Im Rahmen dieser Arbeit erfolgt zudem eine Kopplung des nicht-radioaktiven ECD an einen kommerziellen Gaschromatographen zur Demonstration der Funktionsweise als GC-Detektor. Darüber hinaus wird ein gepulster Betrieb zur Verbesserung des linearen Bereichs implementiert und untersucht.
\end{abstract}

Keywords: Elektroneneinfangdetektor,Gaschromatographie,GC-Detektor,nicht-radioaktiver ECD,nicht-radioaktive Elektronenquelle.

\section{Einleitung}

Elektroneneinfangdetektoren (engl. electron capture detector, ECD) werden weithin allein oder in Kombination mit der Gaschromatographie (GC) für den schnellen Nachweis oder die Analyse von elektronenaffinen Substanzen wie Pestiziden, Herbiziden oder Fluorchlorkohlenwasserstoffen verwendet. Mit erreichbaren Detektionsgrenzen im niedrigen $\mathrm{ppt}_{\mathrm{v}}$-Bereich sind Elektroneneinfangdetektoren seit ihrer Erfindung vor mehr als 55 Jahren [1] die wichtigsten Detektoren in der Gaschromatographie zum Nachweis von solchen Verbindungen. Abgesehen von der Einführung des gepulsten Betriebes [2] ist ihr Grundaufbau nahezu unverändert geblieben. Aufgrund ihres Funktionsprinzips werden hochkinetische Elektronen bei Atmosphärendruck benötigt, die üblicherweise unter Verwendung eines radioaktiven $\beta$-Zerfalls erzeugt werden. Diese Elektronen ionisieren zunächst das Trägergas (Sticksoff oder Luft, bei Helium oder Wasserstoff als Trägergas wird Stickstoff oder Luft als Make-up-Gas zugeführt), wodurch letztendlich die erforderlichen thermalisierten Elektronen entstehen. Der Einsatz radioaktiver Strahler führt jedoch zu gesetzlichen Beschränkungen hinsichtlich Anschaffung, Betrieb und Entsorgung. Obwohl bereits viele Versuche unternommen wurden, die radioaktive Quelle zu ersetzen [3], hat nur die gepulste Heliumentladung einen gewissen Grad an Bedeutung gewonnen. In anderen Gebieten, wie beispielsweise in der lonenmobilitätsspektrometrie, wurden hingegen radioaktive Elektronenquellen erfolgreich durch nicht-radioaktive Elektronenquellen ersetzt. In den letzten Jahren wurde an unserem Institut eine kompakte, hermetisch verschlossene, nicht-radioaktive Elektronenquelle entwickelt [4] (siehe Abb. 1), die freie Elektronen durch thermische Emission in einer Vakuumkammer erzeugt. Diese Elektronen werden dann beschleunigt und können teilweise durch eine $300 \mathrm{~nm}$ dünne Siliziumnitrid-Membran $\left(\mathrm{Si}_{3} \mathrm{~N}_{4}\right)$ zu Atmosphärendruck (1013 mbar) transmittieren. Durch Variieren der Beschleunigungsspannung und des Filamentstroms können die Elektronenenergie und die Elektronendichte gesteuert werden. 


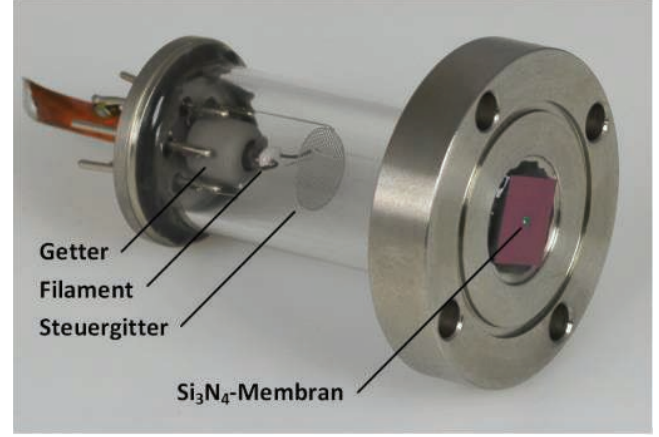

Abb. 1: Foto der nicht-radioaktiven Elektronenquelle mit geheiztem Filament.

Diese Elektronenquelle lässt sich nun an eine kleine lonisationskammer mit einem FaradayDetektor koppeln, wie in Abb. 2 schematisch dargestellt $[5,6]$. Durch Anlegen einer konstanten Kollektorspannung $U_{\text {col }}$ zwischen der Beschleunigungselektrode der Elektronenquelle und dem Faraday-Detektor werden die erzeugten Elektronen in Richtung des Faraday-Detektors bewegt und somit ein konstanter Detektorstrom erzeugt. Das Trägergas mit den darin enthaltenen Analyten strömt vom Faraday-Detektor in Richtung Elektronenquelle. Elektronenaffine Verbindungen fangen nun die freien, thermalisierten Elektronen ein, was zu einem verringerten Detektorstrom als Sensorsignal führt. Der Detektorstrom wird durch einen an unserem Institut entwickelten und angepassten Transimpedanzverstärker [7] verstärkt und von einem Keysight 34461A Multimeter mit einer Mittelungszeit von 200 ms gemessen.

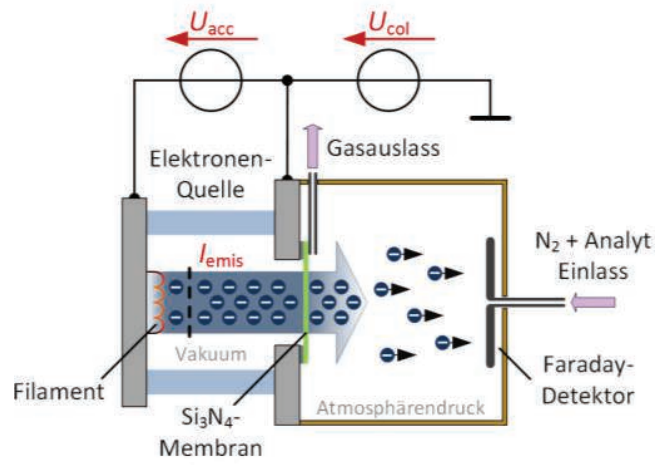

Abb. 2: Schematische Darstellung des Elektroneneinfangdetektors unter Verwendung der nicht-radioaktiven Elektronenquelle aus Abb. 1.

In vorausgegangenen Arbeiten zum nichtradioaktiven ECD [5,6] konnten durch Optimierung der Betriebsparameter unter Verwendung einer DC-Kollektorspannung von $U_{\text {col, DC }}=5 \mathrm{~V}$ bereits Nachweisgrenzen von 850 ppt $_{\mathrm{v}}(0,005 \mu \mathrm{g} / \mathrm{l})$ für 1,1,2-Trichlorethan und $190 \mathrm{ppt}_{\mathrm{v}}(0,002 \mu \mathrm{g} / \mathrm{l})$ für Sevofluran in Stickstoff erreicht werden. Diese Nachweisgrenzen sind vergleichbar mit denen radioaktiver ECDs mit $0,006 \mu \mathrm{g} / \mathrm{l}$ für 1,1,2Trichlorethan [8]. Im nächsten Schritt soll nun dieser nicht-radioaktive ECD an einen kommerziellen Gaschromatographen gekoppelt sowie anhand verschiedener Beispielsubstanzen dessen Eignung als GC-Detektor demonstriert werden. Darüber hinaus erfolgen Untersuchungen zum Betrieb mit gepulster Kollektorspannung, um den linearen Bereich des ECD zu verbessern.

\section{GC-Kopplung}

Der zuvor beschriebene ECD mit nichtradioaktiver Elektronenquelle soll nun an einen Agilent 7890A Gaschromatographen gekoppelt werden. Dazu war zunächst eine konstruktive Überarbeitung der lonisationskammer erforderlich, um das Volumen des ECD zu verkleinern und damit an die Trägergasflussrate des GC anzupassen. Zudem wurde hierbei basierend auf den vorangegangenen Arbeiten die Gasströmung weiter optimiert, wodurch die Analyten besser in den Bereich der thermalisierten Elektronen geleitet sowie die gebildeten Analytionen besser abtransportiert werden können, um ein verbessertes Signal-zuRausch-Verhältnis zu erreichen.

Für die grundlegende Demonstration des ECD kam zunächst keine spezielle GC-Säule für Pestizide, Herbizide oder Fluorchlorkohlenwasserstoffe zum Einsatz. Stattdessen wurde eine bereits vorhandene Rtx-Volatiles-GCSäule (Restek) mit einer Länge von $30 \mathrm{~m}$, einem Innendurchmesser von $530 \mu \mathrm{m}$ sowie einer Schichtdicke von $2 \mu \mathrm{m}$ verwendet. Als Trägergas für die GC-Säule kommt gereinigter Stickstoff mit einem konstanten Volumenstrom von $25 \mathrm{mls} / \mathrm{min}$ zum Einsatz. Dieser ist verhältnismäßig hoch gewählt, um einen Kompromiss aus den in den vorausgegangenen Arbeiten optimierten Parametern für den ECD [5] und einer ausreichenden Trennleistung des GC zu gewährleisten. Durch Optimierung der Steuerungselektronik der Elektronenquelle konnte der Emissionsstrom von $40 \mathrm{nA}$ [6] auf $50 \mathrm{nA}$ erhöht werden, um der verringerten Sensitivität durch den geringeren Volumenstrom im ECD entgegenzuwirken. Als Analyten kommen die bereits in den Vorarbeiten $[5,6]$ untersuchten Stoffe Sevofluran mit einer Konzentration von $100 \mathrm{ppbv}$ sowie 1,1,2Trichlorethan mit einer Konzentration von 600 ppbv in Stickstoff zum Einsatz. Zusätzlich erfolgt die Zugabe von weiteren Beispielsubstanzen.

Zur Aufnahme des Gaschromatogramms wird zu Beginn der Messung das Probengas aus 
einer Probenschleife mittels 6-Port-Ventil in das Trägergas injiziert. Die GC-Temperatur bleibt zunächst für $30 \mathrm{~s}$ auf $50{ }^{\circ} \mathrm{C}$ und steigt dann mit einer Rate von $20^{\circ} \mathrm{C} / \mathrm{min}$ auf $90^{\circ} \mathrm{C}$ an. Dadurch wird trotz des hohen Flusses eine ausreichende Trennleistung erreicht. Das zugehörige Gaschromatogramm ist in Abb. 3 dargestellt.

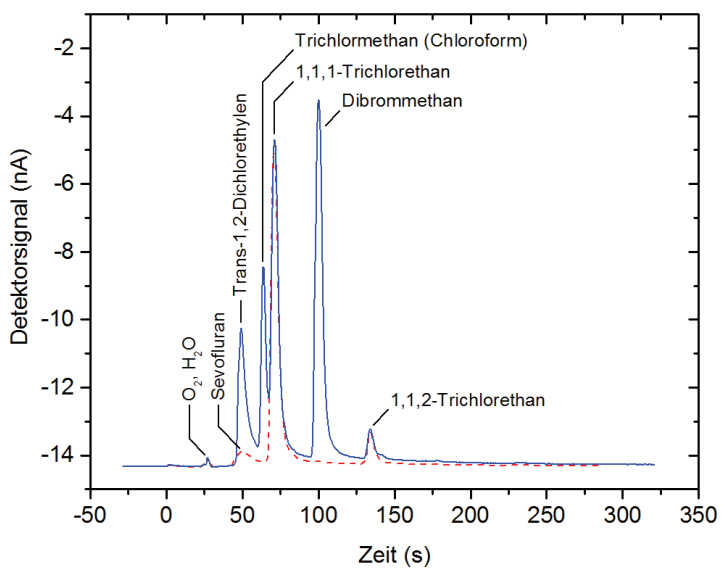

Abb. 3: Gaschromatogramm mit $100 p p b_{v}$ Sevofluran, $\quad 600 p p b_{v} \quad 1,1,2-$ Trichlorethan und 1,1,1-Trichlorethan (rot, gestrichelt) sowie zusätzlich mit anderen Beispielsubstanzen (blau, durchgezogen). Parameter: $25 \mathrm{~m} / \mathrm{s} / \mathrm{min}$ Trägergasfluss; $50{ }^{\circ} \mathrm{C}$ GC-Temperatur, Temperaturrampe nach $30 \mathrm{~s}$ mit $20^{\circ} \mathrm{C} / \mathrm{min}$ auf $90^{\circ} \mathrm{C}$.

Nach etwa $30 \mathrm{~s}$ ist bereits ein erster Peak mit geringer Amplitude im Chromatogramm zu erkennen, der insbesondere von Restsauerstoff und Restfeuchte im Probengas verursacht wird. Während Sevofluran die gleiche Retentionszeit wie Trans-1,2Dichlorethylen aufweist und damit im verwendeten Gasgemisch praktisch nicht erkennbar ist, können die übrigen Substanzen gut voneinander unterschieden werden und demonstrieren somit die grundsätzliche Eignung des entwickelten nicht-radioaktiven ECD als GC-Detektor.

Darüber hinaus wird die erreichbare Nachweisgrenze für Sevofluran und 1,1,2Trichlorethan im GC-Betrieb bestimmt und mit den vorangegangenen Arbeiten verglichen. Die Berechnung erfolgt anhand des gemessenen linearen Anstiegs der Peak-Amplitude bei geringen Konzentrationen. Die Nachweisgrenze wird definiert als dreifache Standardabweichung des Rauschens der Basislinie in einem Bereich ohne Analytpeak im Gaschromatogramm.

Um eine konstante, bekannte Analytkonzentration im Probengas einzustellen, wird der Analyt in ein Permeationsröhrchen gefüllt, das in einem temperaturgeregelten Permeationsofen auf $35^{\circ} \mathrm{C}$ erhitzt wird. Der Ofen wird dabei mit Stickstoff mit einem konstanten Durchfluss von $600 \mathrm{mls} / \mathrm{min}$ gespült. Ein einstellbarer Teil dieses Gases kann nun mit reinem Stickstoff verdünnt werden. Für die folgende Bestimmung der Nachweisgrenze wurden 1,1,2-Trichlorethan (CAS: 79-00-5) und Sevofluran (CAS: 28523-86-6) von Sigma Aldrich verwendet.

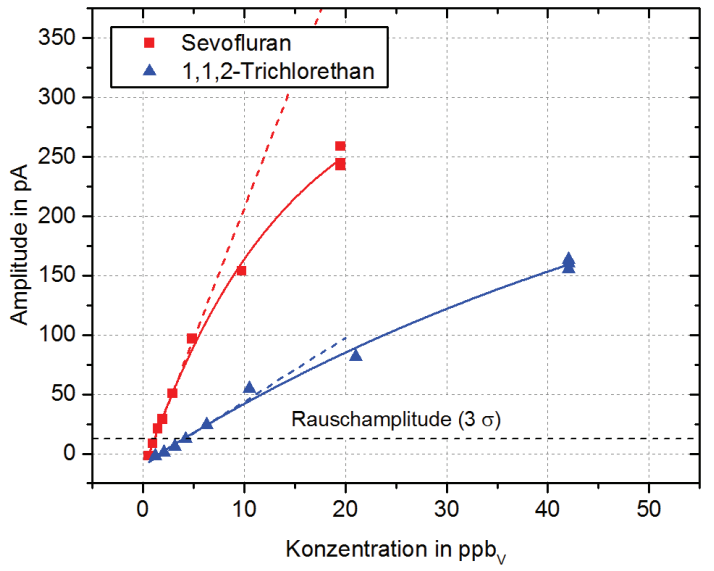

Abb. 4: Kalibrierkurven zur Bestimmung der Nachweisgrenze für Sevofluran und 1,1,2-Trichlorethan. Parameter: $25 \mathrm{~m} / \mathrm{s} / \mathrm{min}$ Trägergasfluss, konstant $80^{\circ} \mathrm{C}$ GC-Temperatur.

Abb. 4 zeigt die resultierenden Kalibrierkurven für die beiden Analyten. Hieraus ergeben sich die Nachweisgrenzen im GC-Betrieb zu 2,4 ppbv (14,4 ng/l) für 1,1,2-Trichlorethan und $0,6 \mathrm{ppbv}(5,3 \mathrm{ng} / \mathrm{l})$ für Sevofluran. Diese Werte sind lediglich um den Faktor 2 größer als im kontinuierlichen Betrieb ohne vorgeschalteten Gaschromatographen [5]. Diese Abnahme lässt sich vor allem durch die Verbreiterung und die damit einhergehende reduzierte Amplitude der Analytpeaks aufgrund der nicht optimalen GC-Säule, eines nicht optimalen Volumenstroms durch den GC sowie eines nicht optimalen Volumenstroms durch den ECD erklären.

\section{Gepulster Betrieb}

Wie bereits in Abb. 4 zu sehen, ist die typische nichtlineare Signalantwort von ECDs im DCBetrieb bei zunehmender Konzentration deutlich sichtbar [2,9]. Daher erfolgt nun die Implementation des aus der Literatur bekannten Betriebs mit gepulster Kollektorspannung, um den linearen Bereich des Detektors zu vergrößern [2,9-11]. Im gepulsten Betrieb werden die Elektronen im Reaktionsbereich überwiegend unter feldfreien Bedingungen erzeugt, während die Analytmoleküle mit den 
Elektronen reagieren und die Analytionen im Gasstrom entfernt werden. Ein kurzer Spannungspuls erzeugt dann im Reaktionsbereich ein transientes elektrisches Feld und die verbleibenden Elektronen werden zum Faraday-Detektor beschleunigt. Der Spannungsimpuls muss dabei so kurz sein (typischerweise einige Mikrosekunden oder noch kürzer), dass die geladenen Analytmoleküle mit ihrer geringeren Beweglichkeit den Detektor in dieser Zeit nicht erreichen [10]. Die Amplitude dieses Spannungsimpulses ist typischerweise deutlich höher als im DC-Modus, aber in der Literatur nicht genau beschrieben. Die Amplitude wird daher auf $U_{\text {col,Puls }}=80 \mathrm{~V}$ eingestellt und die Periodendauer bzw. die Pulsfrequenz so geregelt, dass sich ein konstanter Detektorstrom unabhängig von der Analytkonzentration einstellt. Die erforderliche Pulsfrequenz ist nun ein Maß für die Analytkonzentration.

Erste Messungen wurden mit einer großen Pulsweite von $5 \mu \mathrm{s}$, einem konstanten Detektorstrom von 7,7 nA und einem konstanten Emissionsstrom von lemis $=40 \mathrm{nA}$ durchgeführt, um etwa in der Mitte des möglichen Eingangsstrombereichs des Transimpedanzverstärkers zu bleiben. Abb. 5 zeigt exemplarisch die Kalibrierkurve von 1,1,2-Trichlorethan im gepulsten Betrieb.

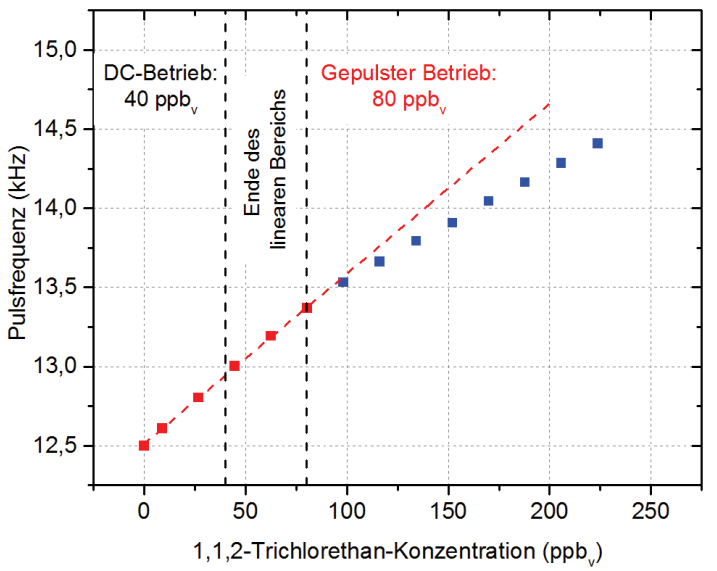

Abb. 5: Erweiterter linearer Bereich durch Verwendung einer gepulsten Kollektorspannung mit $U_{\text {col,Puls }}=80 \mathrm{~V}$ und einer Pulsbreite von $5 \mu$ s.

Die Verwendung der gepulsten Kollektorspannung führt zu einer Verbesserung des linearen Bereichs von $5 \cdot 10^{1}$ auf $10^{2}$ unter Verwendung des genannten Parametersatzes, siehe Abb. 5. Dieser lineare Bereich von $10^{2}$ ist lediglich eine Verbesserung um den Faktor Zwei gegenüber dem linearen Bereich des DC-Modus. Weitere Verbesserungen, wie beispielsweise eine optimierte Regelelektronik für kürzere Pulsbreiten, sollten den erzielbaren linearen
Bereich bis etwa $10^{4}$ erweitern können, der typischerweise mit handelsüblichen ECDs mit radioaktiver Elektronenquelle erreicht wird [10].

\section{Fazit}

In vorhergehenden Arbeiten wurde ein nichtradioaktiver ECD, bestehend aus einer Ionisationskammer sowie einer nicht-radioaktiven Elektronenquelle, aufgebaut und anhand zahlreicher Untersuchungen hinsichtlich der erreichbaren analytischen Leistungsfähigkeit durch Anpassung verschiedenster Betriebsparameter optimiert. In dieser Arbeit erfolgte nun erstmals eine Kopplung dieses ECD an einen kommerziellen Gaschromatographen zur Demonstration der Eignung als GC-Detektor. Für diese ersten GC-Untersuchungen wurde eine bereits vorhandene GC-Säule verwendet, die für die untersuchten Substanzen nicht optimal ist. Für erste Messungen wurde der Fluss durch die Säule und damit durch den ECD so gewählt, dass sich ein Kompromiss zwischen erreichbarer Nachweisgrenze und Trennleistung ergibt. Die gewählten Beispielsubstanzen, insbesondere chlorierte Verbindungen, können leicht in die Gasphase gebracht, im GC getrennt und anschließend zeitlich nacheinander vom ECD detektiert werden. Diese ersten Messungen demonstrieren die Eignung unseres nichtradioaktiven ECD als GC-Detektor für elektronenaffine Substanzen. Anhand der ermittelten Nachweisgrenzen mit 2,4 ppbv $(14,4 \mathrm{ng} / \mathrm{l})$ für $1,1,2-$-Trichlorethan und $0,6 \mathrm{ppbv}$ (5,3 ng/l) für Sevofluran wurde auch die erreichbare Nachweisgrenze durch den gewählten Kompromiss nur geringfügig verschlechtert. In weiterführenden Arbeiten soll eine Charakterisierung mit einer für chlorierte Substanzen angepassten Trennsäule erfolgen. Darüber hinaus ist eine Optimierung der lonisationskammer des ECD erforderlich, um beispielsweise mit einem zusätzlich zugeführten Make-up-Gas die Volumenströme durch den GC und den ECD auf das jeweilige Optimum einzustellen, sodass sich die Trennleistung des GC signifikant verbessert. Verringerte Peakbreiten führen außerdem zu höheren Amplituden und können somit auch die erreichbaren Nachweisgrenzen verbessern.

Darüber hinaus wurde ein gepulster Betrieb zur Verbesserung des linearen Bereichs des Messsignals implementiert und untersucht. Hierbei ergab sich in ersten Experimenten eine Verdopplung des linearen Bereichs. Mit einer speziell für diese Anwendung entwickelten Elektronik ist ein zu kommerziell erhältlichen ECDs mit radioaktiven Quellen vergleichbarer linearer Bereich von etwa $10^{4}$ mit dem in 
dieser Arbeit vorgestellten Aufbau zu erwarten, beispielsweise durch deutlich geringere Pulsbreiten.

\section{Literaturnachweis}

[1] J. E. Lovelock, S. R. Lipsky, J. Am. Chem. Soc. 82, 431-433 (1960); doi: 10.1021/ja01487a045.

[2] R. J. Maggs, P. L. Joynes, A. J. Davies, J. E. Lovelock, Anal. Chem. 43, 1966-1971 (1971); doi: 10.1021/ac60308a014.

[3] W. E. Wentworth, E. D. D'Sa, H. Cai, S. Stearns, J. Chromatogr. Sci. 30, 478-485 (1992); doi: 10.1093/chromsci/30.12.478.

[4] P. Cochems, A. T. Kirk, E. Bunert, M. Runge, P. Goncalves, S. Zimmermann, Rev. Sci. Instrum. 86, 65102 (2015); doi:

10.1063/1.4921707.

[5] E. Bunert, A. T. Kirk, J. Oermann, S. Zimmermann, Electron Capture Detector with Non-Radioactive Electron Source, in: Proceedings, Paris, mdpi, 2017, p. 443.

[6] E. Bunert, A. T. Kirk, J. Oermann, S Zimmermann, An Electron Capture Detector Based on a Non-Radioactive Electron Source. P3.3 - AMA Service GmbH, VonMünchhausen-Str. 49, 31515 Wunstorf, Germany, in: Proceedings of the AMA Conferences 2017 with SENSOR and IRS': SENSOR 2017. 18th International Conference on Sensors and Measurement Technology, Nürnberg, AMA Service GmbH, Wunstorf, 2017, 627-631.

[7] P. Cochems, A. T. Kirk, S. Zimmermann, Rev. Sci. Instrum. 85, 124703 (2014); doi: 10.1063/1.4902854.

[8] F. Barani, N. Dell'Amico, L. Griffone, M. Santoro, C. Tarabella, Journal of Chromatographic Science 44, 625-630 (2006); doi: 10.1093/chromsci/44.10.625.

[9] A. Zlatkis, C.F. Poole, Electron capture: Theory and practice in chromatography, Elsevier Scientific Pub. Co, Amsterdam, New York, 1981.

[10] E13 Committee, Practice for Use of ElectronCapture Detectors in Gas Chromatography, ASTM International, West Conshohocken, PA, 2011.

[11] P. L. Gobby, E. P. Grimsrud, S. W. Warden, Anal. Chem. 52, 473-482 (1980); doi: 10.1021/ac50053a023. 